\title{
Recent Results on Giant Dipole Resonance Decays in Highly Excited Nuclei
}

\author{
Kunt A. Snover \\ Nuclear Physics Lab GL-10 \\ University of Washington \\ Seattle, WA 98195 USA
}

\section{Introduction}

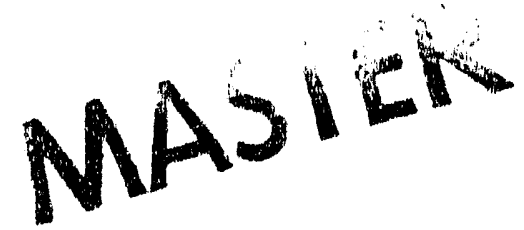

In this paper I discuss some recent results on Giant Dipole Resonance (GDR) decays in highly excited, equilibrated nuclei, based primarily on work done at Seattle. In each of the following four sections I focus on a different topic: namely, oblate shapes of rotating, highly excited Zl - Mo nuclei, adiabatic versus 'motionally narrowed GDR decay, large spin-driven deformations observed in hot medium mass nuclei, and search for entrance channel offects in GDR decay following ${ }^{58} \mathrm{Ni}$ $+{ }^{92} \mathrm{Zr}$ fusion.

\section{Oblate Shapes of Rotating, Highly Excited Zr - Mo Nuclei}

Recently the spectral shapes and angular distributions of high energy gamma rays from the decay of the GDR were measured for the decay of highly excited ${ }^{90} \mathrm{Zr}$ and ${ }^{92} \mathrm{Mo}$ compound nucle in the temperature and spin ranges $1.62 .0 \mathrm{MeV}$ and $0-50 \%$, respectively. At these temperatures, shell efferts should have mostly dissolved away. and the nuclear shape should be well-approximated as that of a rotating liquid drop. In order to examine the evolution of the nuclear shape with angular momentum, three different reactions were studied. forming the compound nticleus at average spins of 9,22 and $33 \hbar$, respectively.

The measured spectral shapes, analyzed using the (ascade statistical model code, all show strongly broadened GDR shapes, with values for the GDR FWHM (full-width at half-maximum) in the range $8-12 \mathrm{MeV}$. due mainly (see below) to the effect of thermal shape fluctuations. The angular anisotropy ( $a_{2}$ coefficient) was found to be very small for the lowest spin case, as expected for a nearly spherical nucleus. For the higher spins, the anisotropy is larger and increases with spin, as expected for a rotating liquid drop which becomes more deformed with increasing spin.

Experiment alone cannot tell us the sense of the deformation, oblate (noncollective) or prolate (collective). Also, although the magnitude of the angular anisotropy is closely rolated to the magnitude of the equilibrium deformation, it is

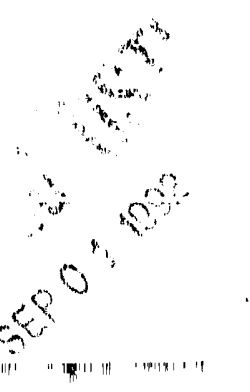


also affected by the shape fluctuations, so that a quantitative analysis requires a detailed theory. In fact, this experiment was designed as a test of one's ability to understand quantitatively both the spectrum shape and the angular distributions from GDR decay in a nuclear system for which the shape; i.e, the potential energy surface (PES) as a function of deformation, is expected to be simple and unambiguous.

Theoretical calculations of the measured spectral shapes and angular distributions have been performed by Alhassid and Bush ${ }^{2}$. Free energy (potential energy) surfaces calculated using the Nilsson-Strutinsky method exhibit minima at deformations $\beta_{0}=0.02,0.08$, and 0.16 at 9,22 and $33 \hbar$, respectively. The minima. lie along the oblate (non-collective) axis, and the values of $\beta_{0}$ are the same as those calculated from the rotating liquid drop model (RLDM) ${ }^{3}$, confirming the lack of importance of shell corrections to the PES at these temperatures. The deformation averaging calculations were done in the adiabatic limit with the metric $\beta^{4}|\sin 3 \gamma| d \beta d \gamma d \Omega$ where $d \Omega=\sin \theta d \theta d \phi$ represents the phase space associated with the Euler orientation angles. The results describe well both the measured spectrum shapes and the $a_{2}$ coefficients. From this good agreement we conclude that both the equilibrium deformations and the curvatures of the PES are given correctly, as well as the essential elements of the (arliabatic) deformation averaging theory. The logic here is that even though the calculated observables depend on several ingredients, it would be unlikely for two or more important elements of the theory to be incorrect and to conspire in such a way as to preserve the good agreement between theory and experiment.

Several other interesting observations follow:

1. The fluctuations are very large, corresponding to most probable deformations of $0.24,0.31$, and 0.46 for the 9,22 and 337 cases, respectively.

2. The apparent splitting in the spectrum shapes for which $E_{2} / E_{1}-1=0.2$ to 0.3 , as deduced from Cascade 2-Lorentzian fits, is larger than can be explained by the (small) equilibrium deformations. The fact that measured and calculated spectral shapes agree very well implies that these apparent splittings are predominately due to the fluctuations.

3. Given that one has orientation fluctuations as in the theory of Alhassid, the observation of a significant angular anisotropy as in the present cases implies that internal shape fluctuations must be very large. This follows because the calculated orientation fluctuations of a fixed shape with $\beta \approx \beta_{0}$ are so large that the angular distribution is very strongly attenuated and it ends up nearly isotropic. That the onentation attenuation should be so large for a fixed shape with $\beta \sim 0.1$ can be easily verified by remembering that the orientation fluctuations re governed by the factor $\exp \left[-\delta E_{\text {rot }} / T\right]$ where $\delta E_{\text {rot }}$ is the difference in rotational energies for the spin aligned parallel and 
perpendicular to the nuclear symmetry axis. The moments of inertia for rotation parallel and perpendicular to the symmetry axis are not very different for $\beta \sim 0.1$, so that for moderate spin, $\delta E_{\text {rot }}$ is small and hence the orientation fluctuations are large. What happens, then, is that the internal nuclear shape fluctuates to very large $\beta$, for which the orientation fluctuations are much smaller (since $\delta E_{\text {rot }}$ is much larger) and a finite anisotropy survives. Even so, the anisotropy would vanish if the internal shape fluctuations averaged over all $\gamma$ equally. But the internal shape always fluctuates about the equilibrimm shape, so that $\gamma=\gamma_{0}$ is favored, and the resulting anisotropy is nonzero. and depends strongly on $\beta_{0 .}$.

4. Given that the theoretical calculations were performed in the adiabatic limit, we conclude there is no significant evidence for motional narrowing from these data. Attempts to interpret 10-20\% differences in measured and calculated $a_{2}$ values in terms of motional narrowing are, in my opinion, unwarranted. There may well be inaccuracies of this size in the adiabatic theory; in addition, a proper average of the calculations over spin and temperature has not been carried out.

In conclusion, the good agreement between measured and calculated spectral shapes and angular distributions in these reactions gives interesting insights into the behavior of hot nuclei. A corollary is that in order to interpret measurements such as these in terms of physically interesting variables such as ecuilibrium deformation, size of fluctuations, etc, requires comparison with reliable. detailed theoretical calculations. 'This is, for the experimentalist, mortunate.

\section{The Timescale for Thermal Shape Fluctuations}

Most data from GDR decay of excited nuclei with temperatures T $\sim 2 \mathrm{MeV}$ or less are well-described by the adiabatic fluctuation theory. However, there exist some data for the decay of ${ }^{108} \operatorname{Sn}^{*}$ and ${ }^{112} \operatorname{Sn}^{*}$ at low excitation energies $\sim 50-60 \mathrm{MeV}$, for which the extracted GDR strength functions are narrower than those calculated with the adiabatic theory', and these differences have been interpreted in terms of 'motional narrowing". Motional narrowing requires the timescale for thermal shape fluctuations, which is not known at present. to be comparable or smaller than $\Delta \omega^{-1}$, where $\Delta \omega \sim \hbar / 15 \mathrm{MeV} \approx{ }^{1} \times 10^{-22}$ sec is the CDR frequency spread due to shape fuctuations. In particular. an early Copenhagen measurement ${ }^{5}$ of the decay of ${ }^{108} \mathrm{Sn}^{*}$ at $\mathrm{E}_{x}=51.8 \mathrm{MeV}(\mathrm{T}=1.3 \mathrm{MeV})$ gave a $\mathrm{FWHM}=6.0 \pm 0.7$ $\mathrm{MeV}$, while an adiabatic calculation by Ormand et $a l^{6}{ }^{\circ}$ yielded a FWHM $=7.8$ MeV. Similarly a Stony Brook measurement gave a FWHM $=6.7 \pm 0.4 \mathrm{MeV}$ for the decay of ${ }^{112 \mathrm{Sn}}$ at $\mathrm{E}_{r}=62.2 \mathrm{MeV}(\mathrm{T}=1.5 \mathrm{MeV})$, to which Alhassid and 
Bush $^{8}$ compared their adiabatic calculation which yielded a FWHM again of 7.8 $\mathrm{MeV}$. However, this calculation was done at the incorrect temperature $\mathrm{T}=1.8$ $\mathrm{MeV}$, which was the (mistaken) value quoted in ref. $T$ (here T always refers to the average final-state temperature calculated accounting for GDR $\gamma-$-emission and for the average energy lost by particle evaporation prior to GDR $\gamma$-emission). On the other hand, there exist earlier Seattle data ${ }^{9}$ for the decay of ${ }^{108} \mathrm{Sn}^{*}$ at $44 \mathrm{MeV}$ (T) $=1.3 \mathrm{MeV}$ ), for which FWHM $=7.3 \pm 0.4 \mathrm{MeV}$, and decay of "itin $\mathrm{Sn}^{*}$ at $48 \mathrm{MeV}$ $(\mathrm{T}=1.4 \mathrm{MeV})$, for which $\mathrm{FWHM}=7.5 \pm 0.4 \mathrm{MeV}$, and these results have been used by Alhassid ${ }^{10}$ as part of the evidence in support of his adiabatic theory.

Contact author for missing photo

Fig. 1. FWHM (full width at half maximum) for (iD) decay in 3 differeit tin isotopes at $\mathrm{T}=1.3 \cdot 1.5 \mathrm{MeV}$. Crosses present result $\mathrm{s}^{11}$ for decay of ${ }^{108} \mathrm{Sn}$ at $\left(\mathrm{E}_{2}, \mathrm{~T}\right)=(50.8 \mathrm{MeV}, 1.35 \mathrm{MeV})$ and ${ }^{112} \mathrm{Sn}_{\mathrm{n}}$ at $(55.8 \mathrm{MeV} .1 .4 \mathrm{MeV}) ;$ open squares earlier Seattle results ${ }^{9}$ for ${ }^{108} \mathrm{Sn}$ at $(44 \mathrm{MeV}, 1.3 \mathrm{MeV})$ and ${ }^{116} \mathrm{Sn}$ af $(48 \mathrm{MeV}$, $1.4 \mathrm{MeV})$; open diamonds ${ }^{108} \mathrm{Sn}$ at $(51.8 \mathrm{MeV}, 1.3 \mathrm{MeV})^{5}$ and ${ }^{112} \mathrm{Sn}$ at $(62.2$ $\mathrm{MeV}, 1.5 \mathrm{MeV})^{7}$. Solid circle calculation of Ormand el al. ${ }^{5}$ for ${ }^{108} \mathrm{Sn}$ at $\mathrm{T}=1.3$ $\mathrm{MeV}$.

Because of the importance of having a theory with a minimal number of adjustable parameters. we ${ }^{11}$ have undertaken some additional measurements of GDR decay in ${ }^{108} \mathrm{Sn}$ and ${ }^{112} \mathrm{Sn}$, in order to help clarify this situation. We meastared the decay of ${ }^{108} \mathrm{Sn}^{*}$ at $50.8 \mathrm{MeV}(\mathrm{T}=1.35 \mathrm{MeV})$ and ${ }^{112} \mathrm{Sn}^{*}$ at $5.5 .8 \mathrm{MeV}(\mathrm{T}=1.4$ $\mathrm{MeV}$ ) formed in ${ }^{16} \mathrm{O}+{ }^{92} \mathrm{Mo}$ and ${ }^{16} \mathrm{O}+{ }^{96} \mathrm{Mo}$ respectively, Measurements were made at 5 different angles in the range $40^{\circ} \cdot 140^{\circ}$. Spectral Cascade fits with $a=$ $\mathrm{A} / 8$ have been performed to the data at 3 of these angles: $55^{\circ}, 90^{\circ}$, and $125^{\circ}$. The fitted values of the FWHM obtained from the data at each angle were averaged; 
the (preliminary) results are FWHM $=7.0 \pm 0.3 \mathrm{MeV}$ fo both reactions. All of these results are summarized in Fig. 1. It is clear that the clata for each isotope are consistent within the quoted uncertainties. Also, over the narrow range of excitation energies spanned by these data, there is no evidence for an excitation energy dependence of the width. In making these comparisons, we have ignored spin differences between the various cases. Since the spins are all small, the widths should be dominated by thermal fli:ctuations. All data are consistent with a GDR width $\cong 7.0 \mathrm{MeV}$, independent of isotope. This is narrower than the result of the adiabatic calculation by Ormand, although the discrepancy (now $\sim 10 \%$ ) is less than previously thought, and does not seem sufficient to imply a clear need for motional narrowing. It may, of course, simply indicate some other. (minor) ciefect in the adiabatic theory.

\section{Large Deformations Observed in the Decay of ${ }^{45} \mathrm{Sc}^{*}$ Compound Nuclei}

We ${ }^{12}$ have been studying the GDR decay of rapidly rotating $A=4.5-6.3$ compound nuclei produced in the following 3 reactions: ${ }^{18} \mathrm{O}+{ }^{27} \mathrm{Al} \rightarrow{ }^{45} \mathrm{Sc}^{*} \cdot{ }^{18} \mathrm{O}+{ }^{45} \mathrm{Sc}$ $\rightarrow{ }^{63} \mathrm{Cu}^{*}$ and ${ }^{32} \mathrm{~S}+{ }^{27} \mathrm{Al} \rightarrow{ }^{59} \mathrm{Cu}$. The motivation for these studies stems from the prediction of the rotating liquid drop model (RLDM) ${ }^{3}$ that muclei in this mass region should be able to sustain large equilibium deformations withcut fissioning. Although the attainable angular momenta are smaller in light nuclei than in heavy nuclei, the corresponding rotational frequencies are much larger. Ai an example, at $23.5 \mathrm{~h}$ in ${ }^{45} \mathrm{Sc}$ (see below) the rotation frequency in $\hbar w_{\text {rot }} \sim 2.9 \mathrm{MeV}$, and the rotation parameter $y \sim 0.15$, and both are about a factor of 4 greater than in ${ }^{160} \mathrm{Er}$ at $50 \mathrm{~h}$. Also, as a result of the fact that light nuclei fission less rearlily than heavy nuclei, highly deformed equilibrim shapes are predicted to exist for a range of angular momenta greater than $\ell_{l}$ but below the fission limit. For angular momenta below the traxial break point $\ell_{1}\left(\ell_{l}=29 h\right.$ for $\left.{ }^{45} \mathrm{Sc}\right)$ the liquid drop shape is oblate, with an equilibrium deformation that depends approximately linearly on $y$ (quadratically on angular moniontum), while for higher the shape become triaxial, and then nearly prolate with a rapidly increasing deformation.

Of the 3 reactions mentioned above we have achiered the most interesting results to date with the first one, ${ }^{18} \mathrm{O}+{ }^{27} \mathrm{Al} \rightarrow{ }^{15} \mathrm{Sc}^{*}$, and I present here the results from measurements at 4 different bombaruing energies, $E_{a b}=44.9,72.5 .89 .4$ and $109.6 \mathrm{MeV}$, corresponding to initial compound nuclear excitation energies 50.0 , $66.6,76.7$ and $88.9 \mathrm{MeV}$. GDR decay in these reactions corresponds to average final-state temperatures of $1.7,1.7,1.8$ and $2.1 \mathrm{MeV}$, and average final-state spins of $13,18.5,21.5$ and $23.5 \mathrm{~h}$, respectively. At these high temperatures we can expect that shell effects are dissolved and that the nuclear shape should be given by the 
RLDM. Furthermore, at the highest energy (spin), about $1 / 3$ of the eraporation cross section contributing to GDR $\gamma$-emission lies at angular momenta beyond $\ell_{l}$.

At each of the 4 energies we measured the spectrum shape and the angular distribution. The high energy part of the measured spectra is clean above $\mathrm{E}_{\text {; }}$ $=12 \mathrm{MeV}$, except for the presence of a $15.1 \mathrm{MeV}$ line in the spectra at the 2 higher bombarding energies. Cascade fits to the measured spectral shapes were performed in these cases by excluding the region near $15 \mathrm{MeV}$ in the fitting. A nors-zero $a_{1}$ coefficient associated with this line was found to be consistent with emission from a source moving with an average component of velocity along the beam axis of $\sim 70 \%$ of the projectile velocity; this, together with the Cascade fits to the spectra measured at different angles allowed the $15.1 \mathrm{MeV}$ background to be subtracted. At other energies above $12 \mathrm{MeV}$ the $a_{1}$ coefficient was found to be zero, consistent with statistical emission.

Our results for the average GDR absortion cross section $\sigma$ inferred from the Cascade fits to the spectial shapes, and for the measured $a_{2}$-coefficients are shown in Fig. 2 for each of the 4 cases. Several interesting features are apparent. Below the GDR centroid, $a_{2}$ is relatively large and negative, $\approx-0.2$ for the highest spin cases. Since -0.25 is the limiting value due to deformation splitting, the observed values are indicative of large deformation. The thin solid lines show the thermal averaging calculations of Alhassid and Bush ${ }^{13}$. In these calculations. which were performed for the average spin, the eguilibrium shape is oblate, as expected, with an equilibrim deformation of $0.15,0.20,0.39$ and 0.43 for the 4 cases, respectively. The measured and calculated $l_{2}$ values are in good agreement below the GDR centroid (and above $12 \mathrm{McV}$ ), whereas at higher energies the measured values are negative for the 2 higher spin cases, while the calculated values are positive, and this difference is not understood.

The measured and calculated absorption cross sections are similar fur all 4 cases. Of particular interest is the apparent second peak or shoukler at $\mathrm{E}_{\gamma} \cong 25$ $\mathrm{MeV}$ in the experimental cross section. In principal, this could be due either to very large thermal deformation fluctuations or to a large equilibrium deformation (or both). However, the lowest spin case has nearly the same temperature as the high spin case and does not show a shoulder. Therefore, the shoulders are not due to fluctuations and must instead be due to a large equilibrium defonmation. From the experimental $\sigma$, we then infer an equilibrium deformation in the 2 highest spin cases of $\beta \cong(0.945)^{-1} \ln \left(\mathrm{E}_{2} / \mathrm{E}_{1}\right)=0.5$ for $\mathrm{E}_{2} \cong 2.5 \mathrm{MeV}, \mathrm{E}_{1} \cong 16 \mathrm{MeV}$. This is the largest equilibrium deformation observed to date for a hot nucleus. It is larger than the calculated value of 0.4 ; however, this value is calculated for the average spin. The calculation should be averaged over spin (as is the experimental result). Although such an average has not been done, it is clear that the nonlinear dependence of deformation on angular momentum squared leads one to expect that a larger average deformation will be obtained from a calculation with a proper spin average. 
Contact author for missing photo

Fig. 2. The average GDR absorption cross section $\sigma$ (top panel) and the $a_{2}$ coefficient (bottom panel) for decay of ${ }^{45} \mathrm{Sc}^{*}$ for each of the 4 bombarding energies. The thick solid line in each of the cross section panels is the absorption cruss section $\sigma_{C A S}$ determined from the Cascade fit to the spectrum shape. The data points in these top panels are given by $\sigma_{\gamma}(\exp ) \cdot \sigma_{C A s} / \sigma_{\gamma}(\mathrm{cas})$ where $\sigma_{\gamma}(\mathrm{exp})$ and $\sigma_{\gamma}$ (cas) are the measured and calculated $\gamma$-emission cross sections, respectively. The dashed lines in each of the panels are the calculations of Alhassid and Bush ${ }^{13}$. 


\section{Absence of Entrance Channel Effects in High Energy Gamma Emission in ${ }^{58} \mathrm{Ni}+{ }^{92} \mathbf{Z r}$}

In recent years a number of experiments have shown anomalies in near masssymmetric fusion-evaporation reactions, particularly' ${ }^{64} \mathrm{Ni}+{ }^{92} \mathrm{Zr} \rightarrow{ }^{156} \mathrm{Er}{ }^{*}$ near the Coulomb barrier ${ }^{14}$. The ${ }^{64} \mathrm{Ni}+{ }^{92} \mathrm{Zr}$ reaction shows evidence for a suppression of neutron evaporation relative to statistical model calculations, whereas ${ }^{12} \mathrm{C}+$ ${ }^{144} \mathrm{Sm}$ forming the same compound nucleus at the same excitation energy appears normal. It has been suggesied that these differences may be due to the persistence of large deformation associated with the near mass-symmetric entrance channel, which results in a higher effective yrast line during evaporat'on.

In spite of the fact that there are problems with tis hypothesis (in fact, no simple explanation fits all the experimental observations) it is important to pursue this problem experimentally. One of the most sensitive ways to determine deformation effects in the fusion-evaporation process would be to examine the spectrum shape of high energy gamma rays emitted in the decay of the GDR. Unusually large deformation effects should show up as a splitting of the GDR strength function (a superdeformed shape should have a GDR energy splitting of $\sim 2: 1$, a very large effect!) or, if there is a distribution of unresolved deformations, the result would be an unusually broad strength function. An elevated yrast line should, in addition, show up as a shift in the apparent GDR centroid.

Previously, two different groups have measured high energy $\gamma$-ray spectrum from $\mathrm{Ni}+\mathrm{Zr}$ reactions. Fornal $\mathrm{ct}$ al. ${ }^{15}$ obtained a poor statistics spectrum from ${ }^{64} \mathrm{Ni}+{ }^{92} \mathrm{Zr}$, which appears normal. Thoennessen et al. ${ }^{16}$, on the other hand, obtained a ${ }^{64} \mathrm{Ni}+{ }^{96} \mathrm{Zr}$ spectrum which appears highly anomalous. Compared to the spectrum measural from ${ }^{16} \mathrm{O}+{ }^{144} \mathrm{Nd}$ forming the same compound nuclens at the same excitation energy, ${ }^{160} \mathrm{Er}$ at $53 \mathrm{MeV}$, the ${ }^{64} \mathrm{Ni}+{ }^{96} \mathrm{Zr}$ high energy $\gamma$-ray spectrum shows a inuch broader 'GDR bump' and relatively much more yield at high energies $\mathrm{E}_{\gamma}>15 \mathrm{MeV}$. While the ${ }^{16} \mathrm{O}+{ }^{144} \mathrm{Nd}$ spectrum is a asonably similar to a normal Cascade calculation, the ${ }^{64} \mathrm{Ni}+{ }^{96} \mathrm{Zr}$ spectrum is very different, and different also from calculations with a raised yrast line with a superdeformed GDR splitting. Unfoitunately, the authors did not obtain an absolute normalization (absolute cross section) for these spectra.

In order to pursue this problem further, we ${ }^{17}$ have recently measured the spectrum of high energy gamma rays from $241 \mathrm{MeV}{ }^{58} \mathrm{Ni}+{ }^{92} \mathrm{Zr}$, which forms ${ }^{150} \mathrm{Er}$ * at $57 \mathrm{MeV}$ excitation energy. The inclusive singles cross section was measured with our large NaI spectrometer and is shown in Fig. 3. Also shown in Fig. 3 are Cascade calculations for the 2 processes which dominate the reaction cross section at this near-barrier bombarding energy; namely, fusion-evaporation and deep inelastic scattering. Deep inelastic decay appears to dominate at low $\mathrm{E}_{\gamma}$, while fusion evaporation clearly dominates at high $\mathrm{E}_{\gamma}$. The deep inelastic contribution 
was estimated assuming ${ }^{18,19} \sigma=235 \mathrm{mb}, \mathrm{Q}=-52 \mathrm{MeV}$ and $(\mathrm{E}, \mathrm{J})=20 \mathrm{MeV}$, $4 \hbar$ and $32 \mathrm{MeV}, 6 \hbar$ for ${ }^{58} \mathrm{Ni}$ and ${ }^{92} \mathrm{Zr}$ products, respectively. This Q-value, which is appreciably more negative than the results of either ref. 19 or 20 would imply, was chosen in order to set a conservative upper limit on the deep-inelastic contribution at high $\mathrm{E}_{\gamma}$; the result is $<20 \%$ for $\mathrm{E}_{\gamma}>11 \mathrm{MeV}$. For $\sigma_{f u s}$ we assumed $370 \mathrm{mb}^{18}$ with transmission coefficients suppressed by a factor of 2 relative to the unitary limit, based on results for ${ }^{64} \mathrm{Ni}+{ }^{92} \mathrm{Zr}$ and ${ }^{64} \mathrm{Ni}+{ }^{96} \mathrm{Zr}$ reactions ${ }^{21}$ - the corresponding average fusion $l$-value is $36 \hbar$. The single-Lorentzian GDR parameters were taken from systematics ${ }^{22}$ and are $E_{0}=14,17,18 \mathrm{MeV}, \Gamma=7.5,8.5$, $9.0 \mathrm{MeV}$, and $\mathrm{S}=1.2$ for decay of ${ }^{150} \mathrm{Er}^{*},{ }^{92} \mathrm{Zr}^{*}$ and ${ }^{58} \mathrm{Ni}^{*}$, respectively.

It is instructive that this simple sum of Cascade calculations describes the measured spectrum so well, especially in the high energy region. A similarly good description of the data for $\mathrm{E}_{\gamma} \geq 11 \mathrm{MeV}$ is obtaincl from a lit with the deep inelastic contribution set to zero, which gave $E_{0}=14.4, \Gamma=8.9 \mathrm{MeV}$ and $S=$ 1.7 .

Contact author for missing photo

Fig. 3: The total cross section $\mathrm{d} \sigma / \mathrm{dE}$ obtained from the measured $90^{\circ}$ cross section (multiplied by $4 \pi$ ) for $\gamma$-ray production from $241 \mathrm{MeV}{ }^{58} \mathrm{~N}+{ }^{92} \mathrm{Zr}$. The curves are Cascade calculations; dot dash - decay of the ${ }^{150} \mathrm{Er}^{*}$ compound nucleus; dashed and dotted - decay at ${ }^{58} \mathrm{Ni}^{*}$ and ${ }^{92} \mathrm{Zr}^{*}$, respectively, following deep inelastic scattering; solid-sum of all contributions. 
Unfortunately, S is fairly uncertain; we estimate $\pm 30 \%$ or so. This is based mainly on the uncertainty in the degree of suppression of the fusion transmission coefficients, which have not been measured for ${ }^{58} \mathrm{Ni}+{ }^{92} \mathrm{Zr}$. Thus $\mathrm{S}$ is somewhat high, but not in serious disagreement with ground-state GDR results of $\mathrm{S} \cong 1.2$ in this mass region.

Thus we find that high energy $\gamma$-ray emission in ${ }^{58} \mathrm{Ni}+$ at ${ }^{92} \mathrm{Zr}$ at $\mathrm{E}=241 \mathrm{MeV}$ appears completely normal, with no evidence for an anomalous spectrum shape, a very large strength, or a shifted yrast line. In order to address the question of a possible isotope dependence of anomalous effects, we plan similar measurements of ${ }^{64} \mathrm{Ni}+{ }^{92} \mathrm{Zr}$.

\section{REFERENCES}

1. J.H. Gundlach, K.A. Snover, J.A. Behr, C.A. Gossett, M. Kicinska-Habior and K.T. Lesko, Phys. Rev. Let.t. 65, 2523 (1990).

2. Y. Alhassid and B. Bush, Phys. Rev. Lett. 6.5, 2527 (1990).

3. S. Cohen, F. Plasil, and W.J. Swiatecki, Ann. Phys. 82, 557 (1974).

4. W.E. Ormand, F. Camera, A. Bracco, A. Maj, P.F. Bortignon, B. Million, and R.A. Broglia, preprint, 1991.

5. J.J. Gaardhoje, C. Eilegaard, B. Herskind, and S. Steadman, Phys. Rev. Lett. $\underline{53}, 148(1984)$.

6. W.E. Ormand, P.F. Bortignon, R.A. Broglia, T. Dossing, and B. Lauritzen, Phys. Rev. Let.t. 64, 2254 (1990).

7. D.R. Chakrabarty, S. Sen, M. Thoennessen, N. Alamanos, P. Paul, R. Shicker, J. Stachel, and J.J. Gaardhoje, Phys. Rev. C. 36, 1886 (1987).

8. Y. Alhassid and B. Bush, Phys. Rev. Lett. 63, 2452 (1989).

9. C.A. Gossett, J.H. Gundlach, J.A. Behr, G.A. Feldman, M. Kicinska-Habior, and K.A. Snover, as quoted in ref. 22.

10. Y. Alhass:d and B. Bush, Nucl. Phys. A $\underline{509}, 461$ (1990).

11. Z.M. Drebi, K.A. Snover, M.S. Kaplan, and A.W. Charlop, to be published.

12. M. Kicinska-Habior, Z.M. Drebi, J.A. Behr, C.A. Gossett, A.W. Charlop, M.S. Kaplan, D.P. Wells, and K.A. Snover, to be published.

13. Y. Alhassid and B. Bush, private communication. 
14. See e.g., W. Kuhn et al., Phys. Rev. Lett. 51, is58 (1983); A. Ruckelshausen et al., Phys. Rev. Lett 56 , 2356 (1986); R.V.F. Janssens et al., Phys. Lett. $181,16(1986)$.

15. B. Fornal et al., Phys. Rev. C $\underline{42}, 1472$ (1990).

16. M. Thoennessen, J.R. Beene, R.L. Auble, F.E. Bertrand, C. Baktash, M.L. Halbert, D.J. Horen, D.G. Sarantites, and D.W. Stracener, in Proceedings of the Workshop on the Interface between Nuclear Structure and Heavy Ion Dynamics, University of Notre Dame, 1990.

17. M.S. Kaplan, K.A. Snover, Z.M. Drebi, and D.P. Wells, to be published.

18. F. Scarlassara, S. Beghini, F. Soramel, C. Signorini, L. Corradi, G. Montagnoli, D.R. Napoli, A.M. Stefanini, and Zhi-Chang Li, Z. Phys. A 338,171 (1990).

19. F.L.H. Wolfs, Phys. Rev. C $\underline{36}, 1379$ (1987).

20. V.E. Viola, K. Kwiatkowski, and M. Walker, Phys. Rev. C131, 1550 (1985).

21. A.M. Stefanini, L. Corradi, H. Moreno, L. Mueller, D.R. Napoli, P. Spolaore, E. Adamides, S. Beghini, G.F. Segato, F. Soramel and C. Signorini, Phys. Lett. B 252, 43 (1990); W. Kuhn et al., Phys. Rev. Lett. 62, 1103 (1989).

22. K.A. Snover, Ann. Rev. Nucl. Part. Sci. 36, 545 (1986).

\title{
DISCLAIMER
}

\begin{abstract}
This report was prepared as an account of work sponsured by an agency of the United States Government. Neither the United States Government nor any agency thereof, nor any of their employees, makes any warranty, express or implied, or assumes any legal liability or responsibility for the arcuracy, completeness, or usefulness of any information, apparatus, product, or process disclosed, or represents that its use would not infringe privately owned rights. Reference herein to any specific commercial product, process, or service by trade name, trademark, minufacturer, or otherwise does not necessarily constitute or imply its endorsement, recommendation, or favoring by the United States Government or any agency thereof. The views and opinions of authors expressed herein do not necessarily state or reflect those of the United States Government or any agency thereor.
\end{abstract}



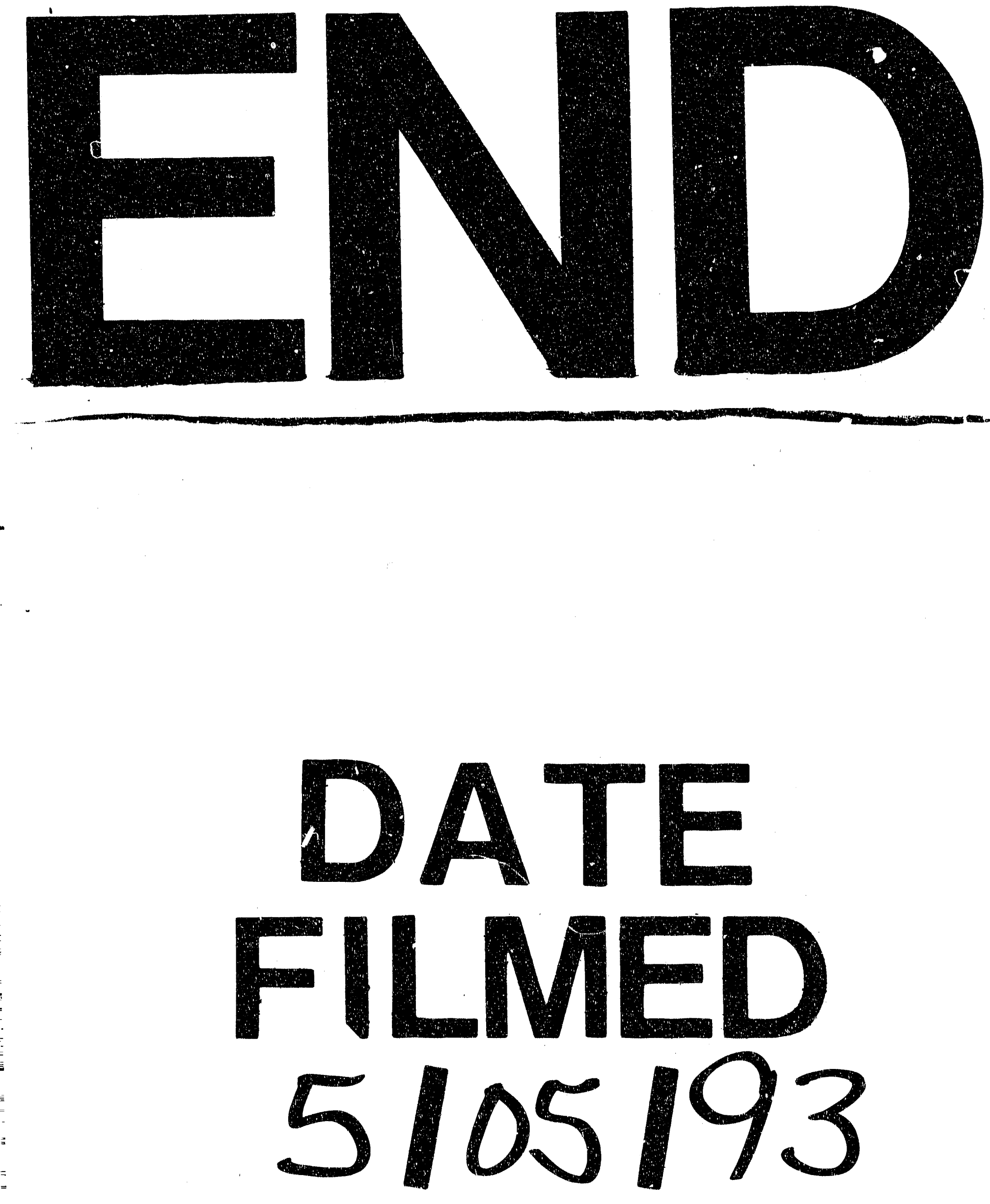